\title{
Caracterización anatómica del desarrollo de la raíz reservante de camotes peruanos (Ipomoea batatas (L.) Lam., Convolvulaceae)
}

\section{Anatomical Characterization of the growth of storage roots of Peruvian Sweet potatoes (Ipomoea batatas, (L.) Lam., Convolvulaceae)}

\author{
M. Marín' y M. Suni
}

\section{RESUMEN}

La caracterización anatómica del desarrollo de la raiz reservante fue llevada a cabo en tres genotipos de camotes. En los estadios iniciales se encontraron diferencias en el desarrollo del xilema primario, incluyendo la formación de un parenquima medular. La actividad cambial secundaria contribuyó en gran medida al desarrollo del parénquima reservante, determinando un arreglo diferencial de ios haces vasculares. Así mismo, fueron hallados granos simples y compuestos de almidón, siendo las dimensiones de los primeros asociadas a las mayores dimensiones celulares del parénquima. Uno de los genotipos evaluados presentó altos valores en el espesor del súber y granos de almidón $(123,9 \mu \mathrm{m}$ y $29,5 \mu \mathrm{m}$ respectivamente). Se sugiere usar estas características como parámetros en la selección de genotipos de camote.

Palabras clave: raiz reservante, cambium secundario, súber, granos de almidón, histología.

\section{ABSTRACT}

The anatomical characterization of the development of the storage root was carried out in three of sweet potato genotypes. In the initial stages differences were found in the development of the primary xylem, including the formation of a medullary parenchyma. The secondary cambial activity contributed to a great extent to the development of the storage parenchyma, determined a differential arrangement of the vascular bundles. Also simple and compound starch grains were found and the dimensions of the first were associated to the bigger size of the parenchyma. One of the genotypes presented high values in the thickness of the phellem and starch grains $\{123,9 \mu \mathrm{m}$ and $29,5 \mu \mathrm{m}$ respectively). These characteristics are suggested as parameters in the selection of sweet potato genotypes.

Key words: storage root, secondary cambia, phellem, starch grains, histology.

\section{INTRODUCCIÓN}

La formación de raíces reservantes en el camote es el resultado de un proceso de engrosamiento que envuelve una serie de eventos anatómicos y fisiológicos desde el temprano desarrollo de la raíz (Wilson, 1982). La formación de la raíz reservante implica la previa diferenciación de las jóvenes raíces adventi-

1 Laboratorio de Anatomia y Farmacognosia Vegetal. Facullad de Ciencras Biologicas. UNiMSM

2 Laboralorio de Fisiologia Vegelal. Facultad de Ciencias Biologicas Universidad Nacional Mayor de San Marcos. Ciudad Universitarla. Av. Venezuela sin, Lima 01, Perú. E-masl. 01900539 unmsm.edu pe cias en raíces gruesas y delgadas. Su formación es mediada por una actividad meristemática secundaria unida a la actividad del cambium vascular. produciendo ambos abundante parénquima de reserva (Hahn and Hozyo, 1983; Bouwkamp and Kays, 1985). Considerando que la síntesis y dimensiones celulares del parénquima reservante y de los granos de almidón duicrminan el engrosamiento de la raíz, el cstudio del componente celular es tan importante como el componente fotosintético y la distribución de la materia seca para el desarrollo de la raíz. 
reservante (Wilson, 1982; Hahn and Hozyo, 1983; Lowe and Wilson 1974; Kays, Magnunson and Fares, 1982).

Los primeros trabajos de Arstchwager (1924) y Togari (Wilson, 1982) establecieron las bases paril los estudios de la raíz reservante en variedades americanas y japonesas de camote respectivamente. Wilson y Lowe (1973) hallaron que la actividad cambial secundaria contribuye tanto como el cambium vascular en la producción del parénquima reservante en las variedades caribeñas de camote. Además se señaló que la caracterización de los granos de almidón, el parénquima reservante y el espesor de la peridermis son los principales aspectos que requieren ser evaluados a nivel histológico, asociándolos a cáracterísticas agronómicas de alto rendimiento y resistencia a plagas (Wilson and Lowe, 1973; Schalk et al., 1986; Noda et al., 1995).

Listudios preliminares llevados a cabo en camotes peruanos mostraron diferencias a nivel histológico en las raíces reservantes en términos de la distribución del sistema vascular secundario (Marin 1994). Considerando que el Perú tiene un número importante de genotipos de camotes, los resultados de la caracterización histológica de estos genotipos podrían usarse en los programas de mejoramiento (Suni y Marín, 1994)

For lo expuesto, el propósito de este trabajo es contribuir al conocimiento del desarrollo de la raíz reservante y la caracterización histológica de tres genotipos peruanos de camote.

\section{MATERIAL Y MÉTODOS}

Esquejes de los genotipos Nacional, Jewel y SR93.440, proporcionados por el departamento de Genética del Centro Internacional de la Papa (CIP) fueron cultivados en la estación experimental de la Molina $\left(12^{\circ} 05^{\prime}\right.$ S, 240 msnm) bajo condiciones normales de cultivo durante el periodo febrero-junio de 1995, en un diseño en bloque completamente aleatorizado con 3 repeticiones, con surcos de $6 \mathrm{~m}$ de longitud y plantas espaciadas cada 30 $\mathrm{cm}$. Se tomaron 7 muestreos durante el periodo de crecimiento, comenzando a us diez días después de plantado (ddp), con una frecuencia de cada diez días hasta el quinto muestreo, luego cada 20 días. Se evaluaron en cada muestreo, nueve plantas por genotipo registrándose el número y diámetro de raíces gruesas y reservantes por planta y procediendo a su evaluación anáómica, para lo cual las raíces muestreadas fueron lavadas y fijadas en Craf III (Johansen, 1940). Las raíces reservantes fueron fijadas en la solución de formaldehido, ácido acético y etanol (FAA); posteriormente fueron almacenadas en etanol $70^{\circ}$

Las raices reservantes fueron seccionadas transversalmente a 20 micras, previo embebido en parafina, y teñidas con la tinción cuádruple de Johansen (Johansen, 1940), luego montadas en bálsamo de Canadá. Las jóvenes raíces gruesas fueron seccionadas a mano, teñidas con cristal violeta y montadas en glicerina fenicada $1 \%$. Para la determinación del engrosamiento de la raíz, las dimensiones celulares del parénquima, granos de almidón y peridermis, se realizaron 15 observaciones microscópicas por variedad. Adicionalmente secciones transversales de las raíces fueron obtenidas reservantes $(1,0 \mathrm{~mm}$ de espesor $)$ para la observación macroscópica de los tejidos

\section{RESULTADOS}

FormaCtón DE RAÍCES ADVENTICIAS Y DESARROLLO PRIMARIO

El desarrollo de las raíces de los genotipos estudiados tuvo características diferenciales; las raices gruesas uvieron estelas de tipo hexarca, pentarca y heptarcas y fueron 
producidas principalmente a partir de primordios radicales localizados en la corteza del tallo (figura 1), próximas a las trazas foliares; las del tipo hexarca fueron las de mayor número. Fueron hallados dos tipos de diferenciación de los tejidos. En estelas de tipo hexarca y heptarca, se presentaron un incompleto desarrollo del xilema con un conspicuo parénquima medular (genotipos Nacional y SR93.440, figura 2). El otro tipo de diferenciación (genotipo Jewel) tuvo un completo desarrollo del xilema, con grandes células metaxilemáticas que ocupaban la zona central del cilindro vascular y escasa formación del parénquima medular.

Adicionalmente ambos tipos presentaron un periciclo, una endodermis definida, espacios esquizógenos en la corteza y una notoria epidermis; así mismo hubo la presencia temprana de laticíferos en el floema primario, drusas de oxalato de calcio la zona cortical y granos de almidón tunto en las células corticales como parénquima medular.

\section{Crecimiento Secundario y COMtenzo del EN- GROSAMIENTO DE IA RAIZZ}

A los 20 dias las raíces gruesas tenían 1,2 mm de diámerro, con presencia del cambium vascular entre los elementos xilemáticos y el comienzo de la proliferación de las células del periciclo.

Las raíce: con un completo desarrollo del xilema tuvieron una estela lignificada, con escasas células parenquimáticas entre los elementos xilemáticos. En este tipo de raíces y en las que presentaron un incompleto desarrolio del xilema, la proliferación de células rodeando a los vasos del metaxilema condujo a una progresiva separación de los elementos xilemáticos en el cilindro vascular por uno de los polos (figura 3).

El engrosamiento de la raíz comenzó a ser notorio hacia la zona proximal o distal a los 30 $\mathrm{ddp}$, cuando las raíces tuvieron un diámetro promedio de $2,21 \mathrm{~mm}$. La actividad del cambium vascular incrementó la expansión de la estela mediante la formación de tejido vascular secundario y principalmente parénquima reservante. En este parénquima se observó los primeros granos de almidón. La continua proliferación de las células del periciclo determinó la formación de la corteza secundaria, y el incremento progresivo en diámetro de la estela hizo evidente el engrosamiento de la raíz (figura 4).

Un típico desarrollo secundario se presentó en las zonas proximal y distal de la raíz. En esta última región la lignificación de la estela fue total (figura 5).

\section{Desarrollo de la raíz reservante}

La producción masiva del parénquima por parte del cambium vascular y cámbiumes secundarios fue continua y uniforme a lo largo de toda la longitud de la raíz. El parénquima reservante producido por el cambium vascular hacia la parte externa de la zona medular (médula externa) tuvo una disposición regular y con células de pequeñas dimensiones en comparación con las células producidas por los cámbiumes secundarios, localizados cin la parte interna de la zona medular (médula intema, tabla 1). La corteza secundaria fue estrecha y generalmente con escasas drusas y granos de almidón. Estos últimos presentaron formas poliédricas y fueron de tipos simple y compuestos, presentando un aspecto caracteristico bajo la luz polarizada (figura 6). El genotipo Nacional presentó los valores más altos en dimensiones celulares del parénquima y granos de almidón (tabla 2).

En el genotipo Nacional, los haces vasculares producidos por los cámbiumes secundarios estuvieron dispersos en toda la médula mientras que en el genotipo Jewel estuvieron concentrados en el centro de la zona medular (figura 8). 
La producción del tejido vascular secundario por parte del cambium vascular presentó un arreglo radial. Adicionalmente se observó la presencia de laticiferos y de drusas dispersas en la zona medular y cortical.

El incremento máximo en longitud de las raíces se alcanzó a los 40 ddp, cuando la pigmentación era la que correspondía a los genotipos. El número de raíces reservantes fue tempranamente determinado (30 ddp), y el genotipo Jewel fue el que presentó el mayor número, y en cuanto a los otros tipos de raíces (fibrosas y tipo lapicero) los tres genotipos los presentaron abundantes (figura 9).

Por otro lado la peridermis fue observada a los $40 \mathrm{ddp}$ presentando un desarrollo variable en su espesor y pigmentación en los genotipos estudiados. El valor más alto en el grosor del súber lo presentó el genotipo $\mathrm{Na}$ cional (tabla 2, figura 7).

\section{DISCUSIÓN}

Inicialmente las raíces del camote se diferenciaron en raíces adventicias, gruesas y delgadas, las últimas de estelas tetrarcas. Las jóvenes raíces gruesas muestran una temprana especialización conducente a su engrosamiento, esto se basa en la presencia de estelas del tipo hexarca, pentarca y heptarcas y a la presencia de una médula parenquimatosa, como lo propuso Togari y Wilson (Wilson, 1973). El desarrollo de estos tipos de estelas permite, conjuntamente con el crecimiento celular, el engrosamiento de la raíz reservante, proceso que es determinado según varios autores, por las auxinas y citoquininas (Matzuo, 1988; Matzuo, Yoneda and Itoo, 1983: ' irace sirjucharran, 1983; Wilson, 1982). Además, el completo desarrollo inicial del xilema primario no impidió el inicio del engrosamiento, como lo demostró la formación de raíces reservantes en el genotipo Jewel.
Las raíces fibrosas se originan de las raíces gruesas y delgadas cuando la temprana lignificación de la esclla impide su engrosamiento. Las diferencias entre las raíces fibrosas y las raíces tipo lapicero se basan principalmente en el desarrol lo de su estela, completamente lignificada en el primero y con un cierto desarrollo del parénquima medular en el segundo, lo cual determina las diferencias en el grosor de estas raíces.

En el caso del genotipo SR93.440, Ias raíces lapicero fueron el principal tipo de raíz producido. Fste resultado contrasta con la buena producción de raíces reservantes que se obtienen en San Ramón (11²08' S, 800 m), una localidad caracterizada por tener tanto altas temperaturas como régimen pluvial (T. Max. $32^{\circ} ; 2000 \mathrm{~mm}$ ), por lo que podemos indicar que el engrosamiento de la raíz reservante puede estar limitado por los factores climáticos (Hahn and Hozyo, 1983; Bouwkamp and Kiiys, 1985).

Aun cuando existen diver:ins tipos de clasificaciones del sistema radical del camote (Wilson, 1982), los resultados que se presentan están de acuerdo con la clasificación propuesta pol Hahn y Hozyo (1983) o la de Bouwkamp y Kays (1985), quienes consideran los tipos de raíces reservantes, lapiceros, fibrosas, gruesas y delgadas, no siendo necesario desde el punto de vista anatómico considerar más tipos de raíces.

La actividad meristemática en el parénquima medular, formando los cámbiumes secundarios, determina la expansión de la estela; posteriormente el desarrollo posterior de la raíz reservante se logra por la actividad combinada del cambium vascular y nuevas zonas cambiales diferenciadas en la médula, ambos produciendo lejido vascular pero principalmente parénquima reservante. Finalmente la actividad cambial secundaria determina un arreglo característico que los diferencia de las variedades caribeñas de camote (figura 8). En 
la figura 10 se plantea un modelo del flujo de cambios histológicos en la formación de la raíz reservante del camote.

Por otro lado, los granos de almidón observados fueron mayormente del tipo simple; sin embargo también se observó el tipo compuesto (no reportado en la literatura). Asimismo se encontró granos de almidón con las dimensiones más grandes que las reportadas para otras variedades (Madamba et al., 1975; Kays, 1992; Liu, S. et al., 1985b; Noda et al., 1995). Los mayores valores en las dimensiones celulares del parénquima y granos de almidón estuvieron asociados con los mayores diámetros de raíces reservantes (tabla 2). Y mientras que Artschwager (1924) señala que el parénquima producido por el cambium vascular es el más rico en almidón, los resultados muestran que principalmente es la actividad cambial secundaria la que da origen a este parénquima, lo que da lugar al desarrollo de la raíz reservante, similar a los resultados hallados por Wilson y Lowe (1973).

Otro aspecto importante son las diferencias encontradas en el grosor de la capa suberosa. Por ejemplo Schalk el al. (1986) indicaron que la resistencia de las variedades de camote al ataque de estadios larvales de insectos del complejo Diabrotica-Systena, está asociado con el mayor engrosamiento de la peridermis en el temprano desarrollo de las raíces; esto también se relaciona con la resistencia al ataque de nemátodes (Lin, He, Wen, Fang and Song, 1996); entonces, las fuertes diferencias en el grosor del súber encontradas (tabla 2) nos indican que este carácter puede usarse para la selección de variedades resistentes al ataque de plagas. Al parecer actuaría como una barrera mecánica aunque también se señala la presencia de constituyentes químicos que estarían contribuyendo a esta resistencia (Lin et al. 1996; Schalk et al. 1986; Ojijo, 1991; Kays, 1992; Clark and Moyer, 1991).

\section{AGRADECIMIENTOS}

Los autores desean expresar su agradecimiento al Ing. Luis Díaz del CLP y a los Profs. Betty Millán y Domingo Iparraguirre, del Laboratorio de Anatomía y Farmacognosia Vegetal de la UNMSM por su invalorable apoyo en la parte experimental, así como a la Dra. Eleucy Pérez por la revisión de la tesis original.

\section{LITERATURA CITADA}

Arstchwager, E. 1924. The anatomy of the sweet potato with notes on internal breakdown. In J. Agric. Res. Washington, D. F., USA.

Bouwkamp, J. and Kays, S. 1985. Sweet potato Products. A Natural Resource for the tropics. Bouwkamp, J. CRC Press-Inc. Eds. Boca Raton, Fl. USA.

Clark, C. A. y Moyer, J. W. 1991. Compendio de enfermedades de la Batata (camote, boniato). Publc. CIP. 96 pp., Lima.

Decheco, E.A. 1993. Evaluación del grado de resistencia relativa de clones de Camote al gorgojo Eucepes postfaccialus (Fairmaire) (Coleoptera: Curculionidae) bajo condiciones de campo y laboratorio. Tesis M. Sc. Publ. CIP. Lima.

Grace Sirju-Charran. 1983. Some biochemical parameters associated with the tuberisation of Sweet potato (İpomoea balatas L. Lam.). In Proc. Caribean Regional Workshop on Tropical Root Crops. Jamaica, Abril 10-16.

Johansen, D.A. 1940. Plant microtechnique. Mc Graw Hill ed., New York. 
Kays, S. J., Magnunson C. E. and Fares, Y. 1982. Assimilation proteins of carbon in developing Sweet potato using ${ }^{11} \mathrm{C}$ y ${ }^{14} \mathrm{C}$. In Sweet Potato Proc. First Int. Symp. R. L. Villarreal and T. D. Geigs (eds.) AVRDC. Taiwan.

Kays, S. J. 1992. The clismical composition of the Sweet potato; $n$ Sweet potato technology for the 21 st. Century. W.A. Hills, C.K. Bonsi (eds.) Tuskegee University, AL. EUA.

Lowe, S. B. and Wilson, L. 1974. Comparative analyses of tuber development in six Sweet potato (Ipomoea batatas L. Lam.) Cultivars. 1. Tuber initiation, tuber growth and partition of assimilates. In Ann. Bot. (London) (N. S.) 38: 307-317.

Liu, S.Y. et al. 1985b. Studies on the physicochemical properties of the tubers of new Sweet potato lines. In Ciunghua nun yeh yen chiu $34: 2$ l-32.

Lin, M., He, L., Wen, L., Fang, Z. and Song, B. 1996. Mechanisms of morphological structure of sweet potato rot nematode (Ditylenchus destructor). In Scientia Agricultura Sinica (China) 1996.29(3):8-12.

Madamba, C. S. et al. 1975. Sweetpotato Starch: Physicochemical properties of the whole starch. In Philipp Agric. 58 (9-10): 338-350.

Marín, Manuel. 1994. Aspectos preliminares de la caracterización histológica de la raíz reservante de Ipomoea batatas L. Lam. En VI Congr. de Jóvenes Científicos. Univ. Cayetano Heredia, I-5 marzo. Lima.

Matzuo, T. 1988. Variations in the levels of major free cylokinins and free abcisic acid during tuber development of sweet potato. In J. Plant Growth Kegulation 7: 249-258.

Matzuo, T., T. Yoneda and S. Itoo. 1983. Identification of free cytokinins and changes in endogenous levels during tuber development of .;weet potato. In Plant Cell. Physiology 24: 1305-1312.

Noda, T., Takahata, Y. Sato, T., Hisamatsu, M., Yamada, Tetsuya. 1995. Physicochemical Properties of Starches Extracted from Sweetporato Roots Differing in Physiological Age. In J. Agric. Food Chem. Vol. 43. N. ${ }^{\circ}$ 2, 1995.

Ojijo, N. K. O. 1991. Objetive Evaluation or Quality Changes in Stored Sweetpotato. Tesis M. Sc. University of Nairobi. Publ. CIP. Lima.

Schalk, J. M., Peterson, J. K., Jones, A., Dukes, P. K., and Walter, W. M. 1986. The anatomy of sweetpotato periderm and relationship to wireworm, Diabrotica y Systena resistance. In I. A gric. Entomolg. (USA) 3 (4) 350-356 pp.

Suni, M. and Marín, M. 1994. Characterization of high yielding clones of Sweetpotatoes. In 10 th. Symposium of the International Society for Tropical Root Crops. ISTRC. Nov. 13 19. Salvador, Bahia. Brasil.

Wilson, L. A. 1982. Tuberization in sweet potato (Ipomoea batatas (L) Lam. In Proc. Fïrst. Uint. Symp. AVRC. China.

Wilson, L. A. and Lowe, S.B. 1973. The anatomy of the rool system in West Indian sweet polato (Ipomoea 
batatas (L.) Lam. Cultivars. Ann. Bot. (Lonion) ns 37: 633-643.

Zuincho R. O. 1990. Inventario de enfermedidur y pligar insecriken el cultivo del Camote (Ipomoea batatas L. Lam.) antes fundo "El Milagro", San Ramón. Tesis Ing. Agron. Univ. Nac. Centro del Perú.

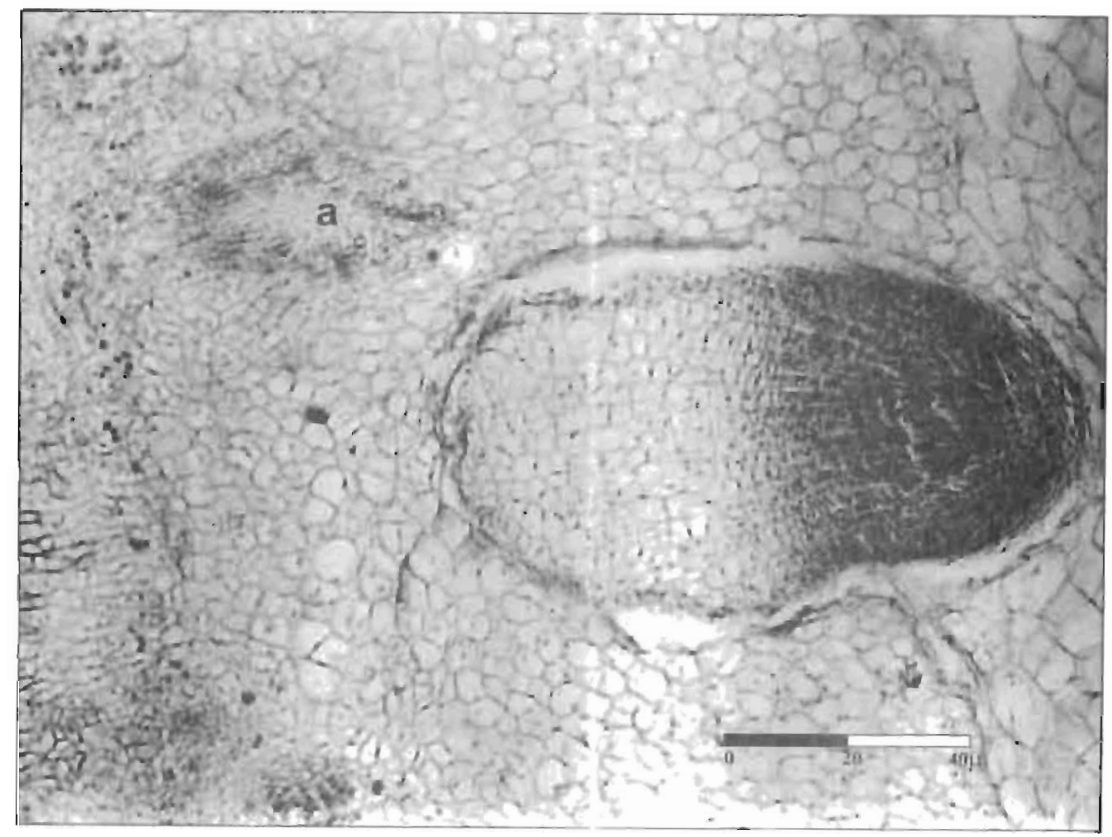

Figura 1. Sección transversal del tallo del gen stipo Jewel mostrando un primordio radical en la zona cortical $32 X$

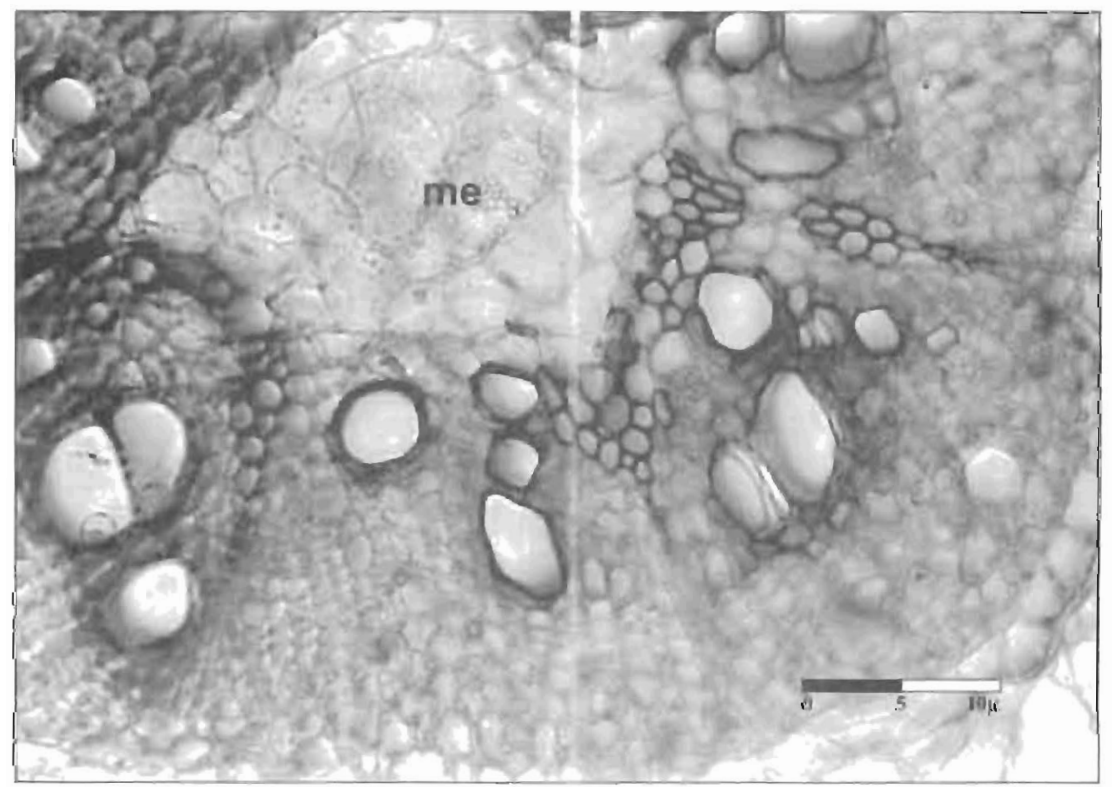

Figura 2. Detalle de la estela secundaria de una raiz gruesa del genotipo SR93.440, mostrando el parénquima medular (me) $100 \mathrm{X}$. 


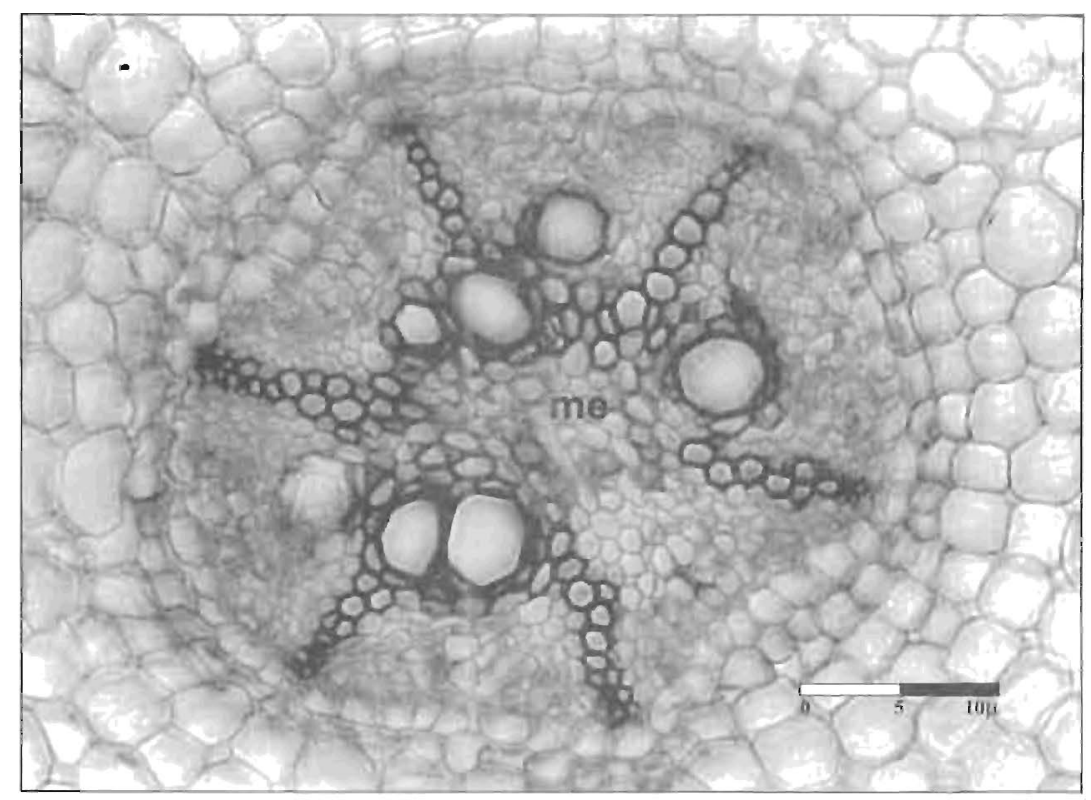

Figura 3. Detalle de una raí:- gruesa del genotipo Nacional (20 ddp). Se observa la proliferación de las células medulares (me) que separa por uno de sus polos al cilindro vascular $100 \mathrm{X}$.

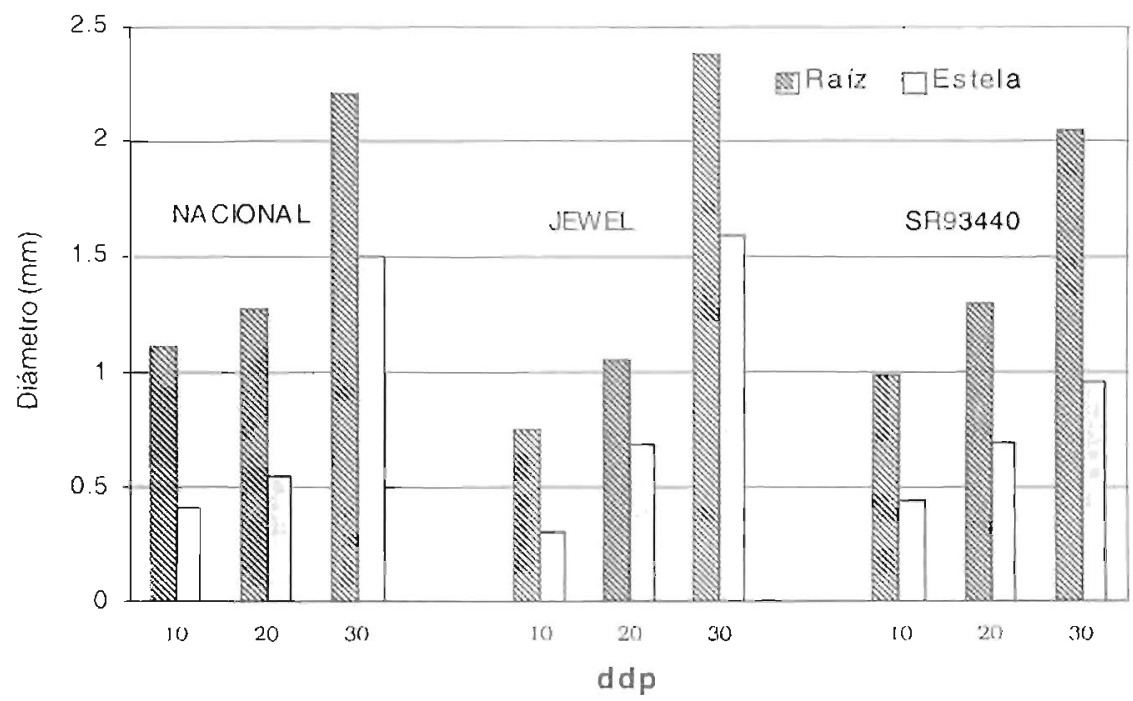

Figura 4. Variación del diámetro de la estela en relación al diámetro de la raíz de los genotipos evaluados durante el primer mes de crecimiento. 


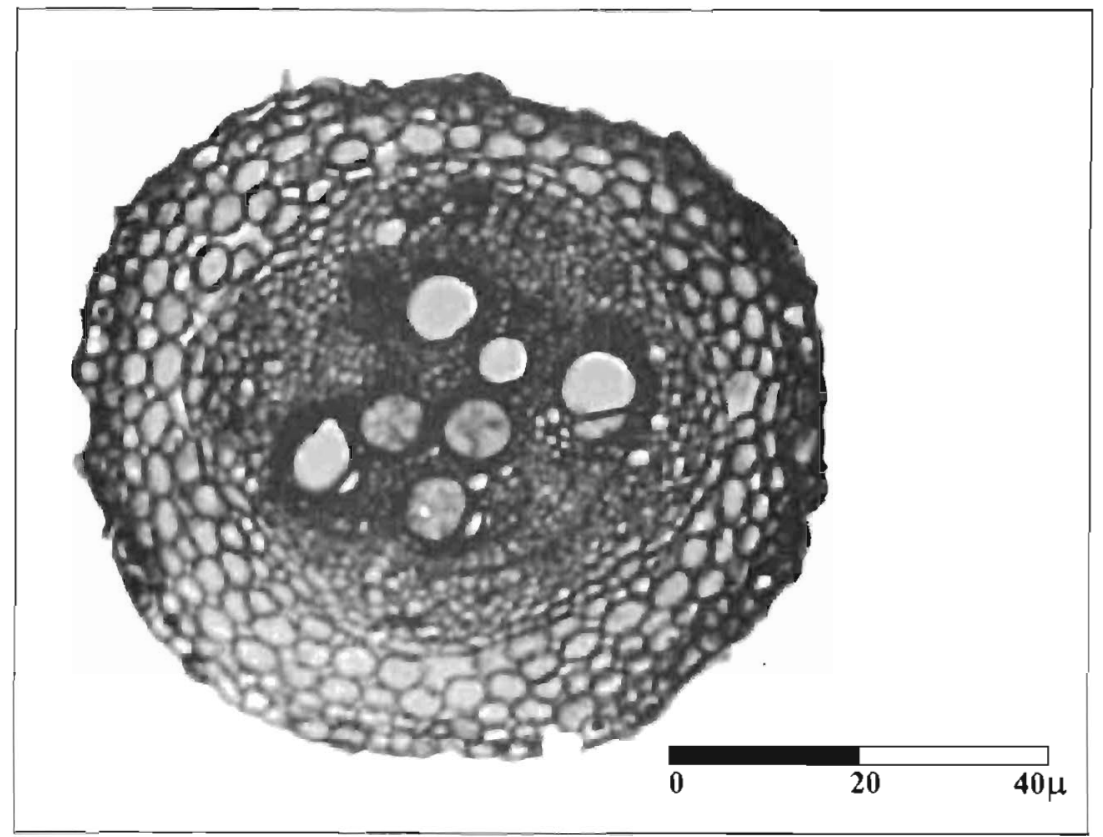

Figura 5. Sección transversal de la parte distal de una raíz gruesa del genotipo jewel (20 dap) mostrando la lignificación total de la estela $32 \mathrm{X}$.

\begin{tabular}{ccccc}
\hline Genolipos & Médula interna $^{\text {a }}$ & hédula externa ${ }^{8}$ & Corteza interna $^{\text {a }}$ & Corteza externa $^{{ }^{a}}$ \\
Nacional & $125.9 \pm 23.6$ & $95.7 \pm 22.0$ & $101.1 \pm 10.5$ & $121.4 \pm 14.8$ \\
Jewel & $114.8 \pm 19.3$ & $74.6 \pm 6.7$ & $95.4 \pm 18.6$ & $120.1 \pm 16.3$ \\
SR93.440 & $107.1 \pm 23.5$ & $99.1 \pm 24.5$ & $72.1 \pm 12.5$ & $107.5 \pm 8.9$ \\
\hline & & &
\end{tabular}

Tabla 1. Dimensiones celulares $(\mu \mathrm{m})$ de células parenquimáticas de raíces reservantes de los genotipos evaluados (90 dap). Los valores del genotipo SR93.440 son referidos a raíces tipo lapicero. 


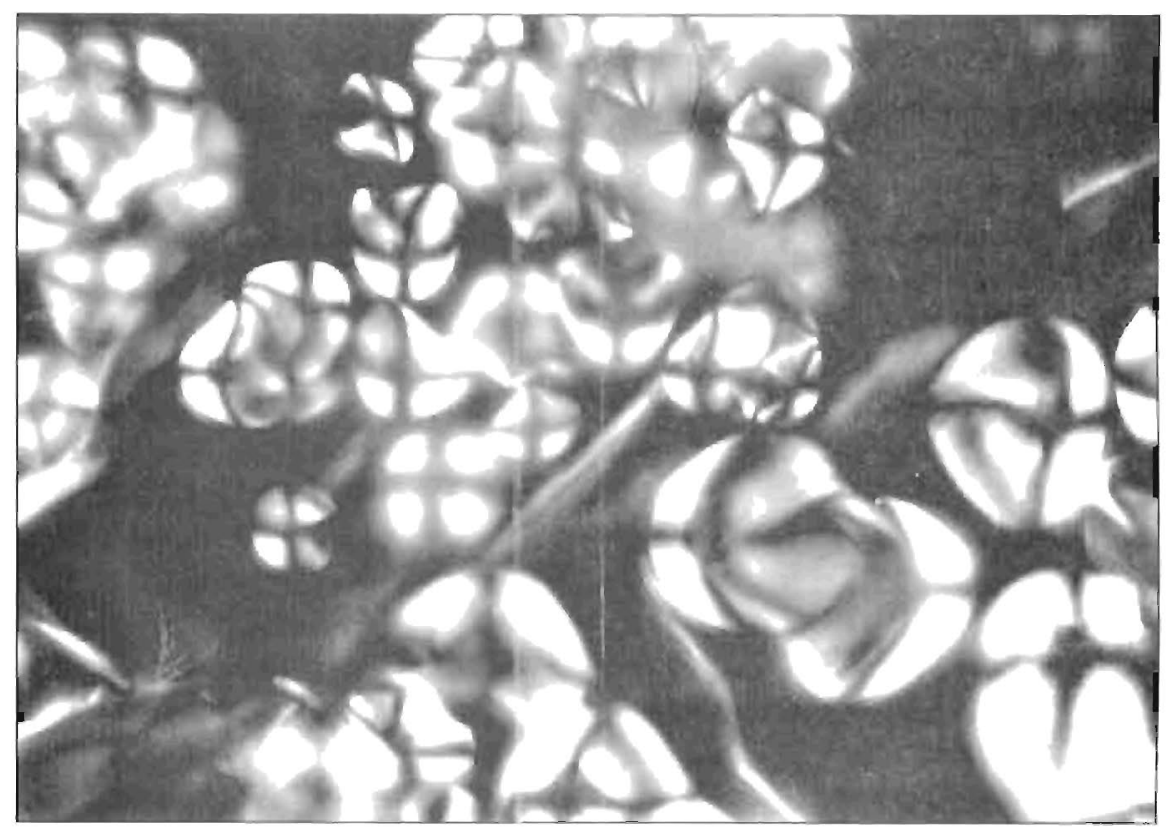

Figura 6. Granos de almidón de la zona cortical próximas al cambium vascular bajo la luz polarizada $900 \mathrm{X}$

\begin{tabular}{ccccc}
\hline Genotipos & $\begin{array}{c}\text { Diámetro de la } \\
\text { raíz reservante } \\
(\mathrm{mm})^{\mathrm{a}}\end{array}$ & $\begin{array}{c}\text { Diánetro de la } \\
\text { capu suberosa } \\
(\mu \mathrm{m})^{\mathrm{a}}\end{array}$ & $\begin{array}{c}\text { Diámetro de } \\
\text { células } \\
\text { medulares }(\mu \mathrm{m})^{\text {a }}\end{array}$ & $\begin{array}{c}\text { Diámetro de } \\
\text { granos de } \\
\text { Almidón }(\mu \mathrm{m})^{\text {a }}\end{array}$ \\
\hline Nacional & $49.5 \pm 7.6$ & $123.9 \pm 9.6$ & $109.5 \pm 26.7$ & $29.5 \pm 8.4$ \\
Jewel & $43.2 \pm 2.5$ & $\$ 16.3 \pm 12.5$ & $96.9 \pm 25.5$ & $12.8 \pm 4.3$ \\
SR93.440 & $8.1 \pm 7.4$ & $94.8 \pm 16.3$ & $102.1 \pm 22.8$ & $22.2 \pm 4.3$ \\
\hline
\end{tabular}

a Promedio de 15 observaciones \pm SD.

Tabla 2. Dimensiones de la raiz y componentes celulares de la raíz reservante de los genotipos evaluados ( $90 \mathrm{ddp}$ ). Los valores del genotipo SR93.440 se refieren a raices tipo lapicero. 


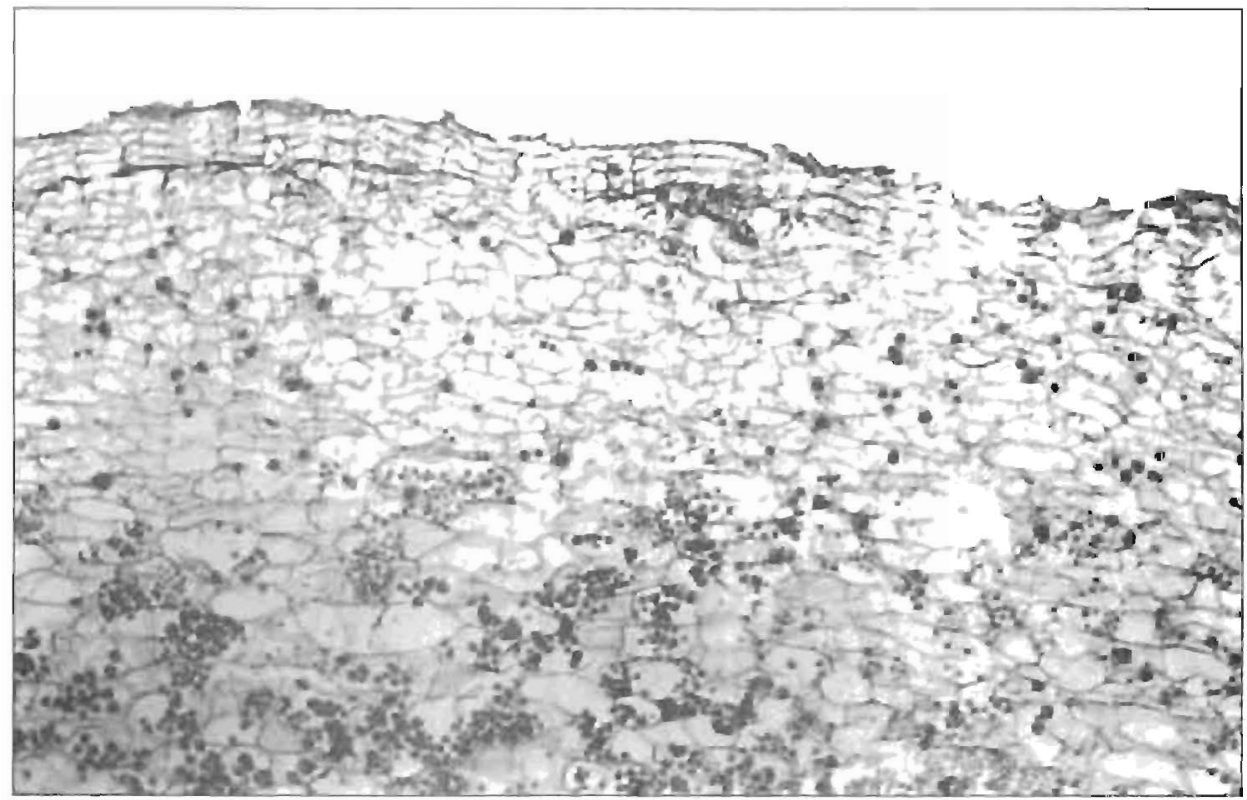

Figura 7. Peridermis $(\mathrm{Pe})$ de la raiz reservante del genotipo Nacional (70 ddp). Nótese el buen espesor de la capa suberosa. Los granos de almidón son abundantes en la zona cortical adyacente al cambium vascular $32 \mathrm{X}$.
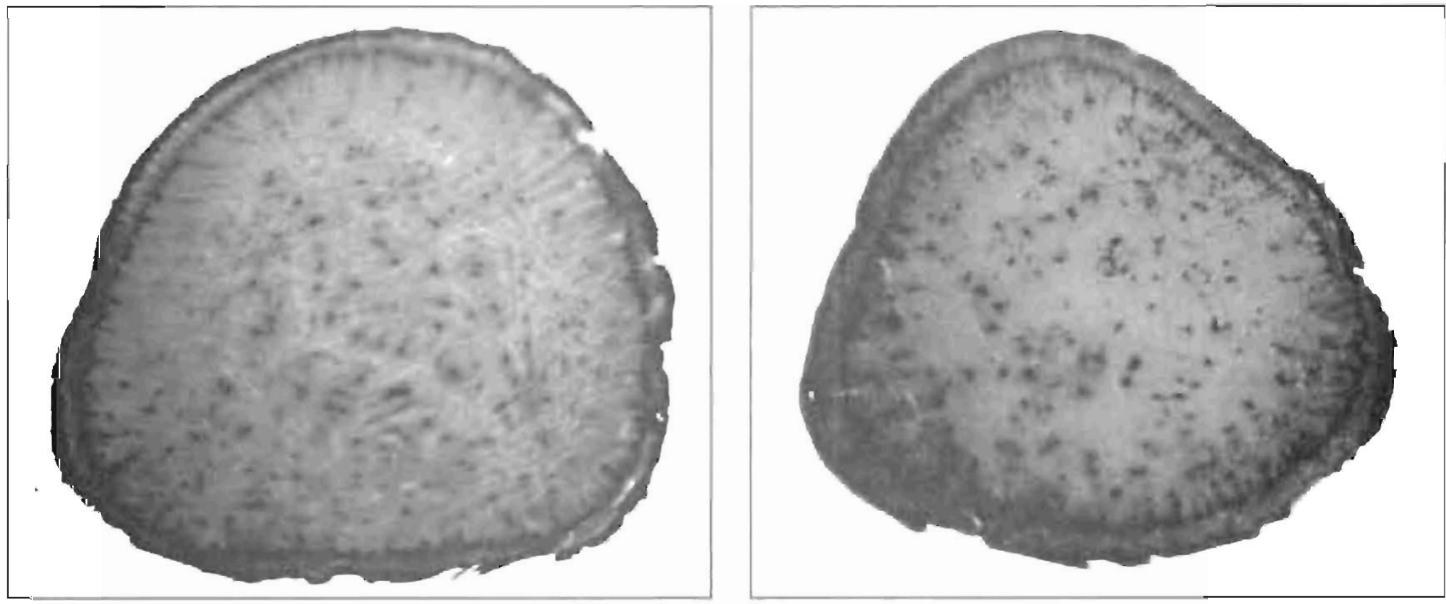

Figura 8. Sección transversal de raices reservantes de los genotipos Jewel (izquierda) y Nacional (derecha), mostrando las diferencias en la distribución de los haces vasculares secundarios. 


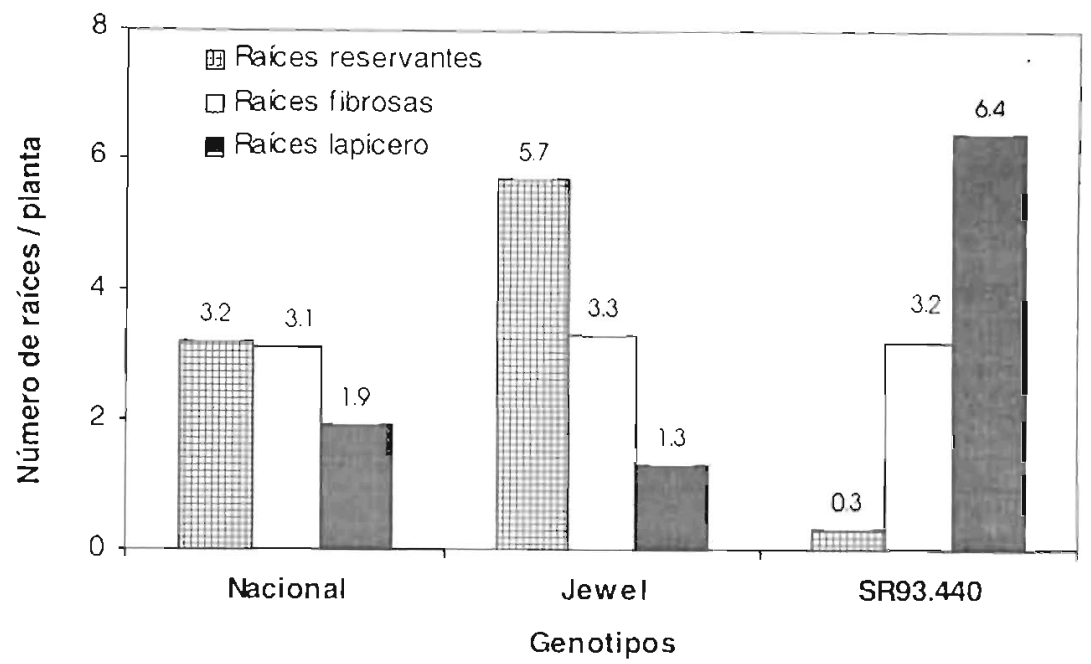

Figura 9. Proporción de los diferentes tipos de raíces producidos por los genotipos evaluados a los $90 \mathrm{ddp}$.

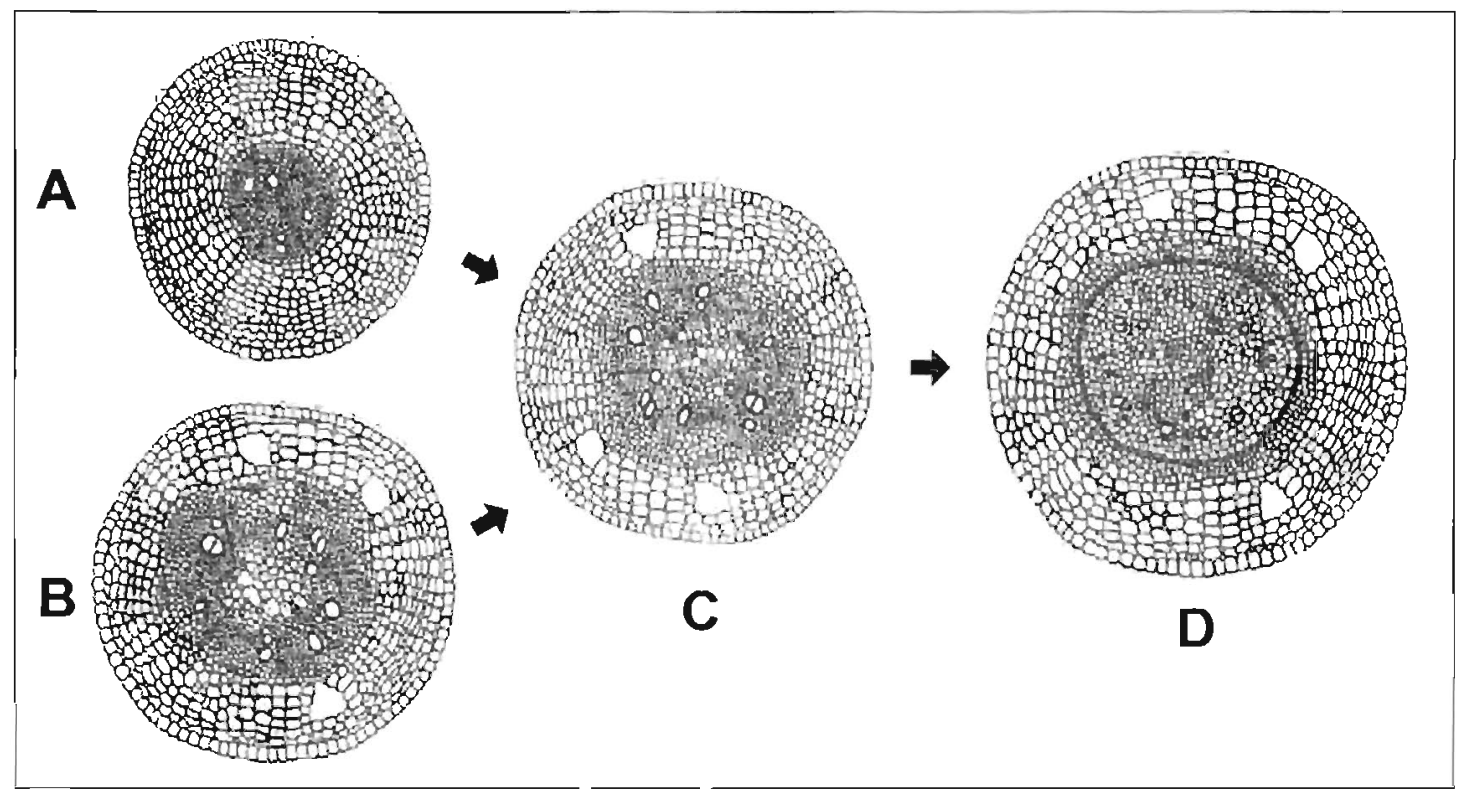

Figura 10. Secuencia histológica del proceso de engrosamiento durante el primer mes de desarrollo. Estructura de la raiz primaria con un completo desarrollo del xilema primario (A), Incompleto desarrollo del xilema primario y formación del parénquima medular (B),10 ddp. Desarrollo secundario: formación del cambium vascular e inicio de la actividad cambial secundaria en la médula (C). Periodo avanzado del engrosamiento (30 ddp): a la actividad del cambium vascular se suma la producción del tejido vascular y parénquima reservante por parte de los cambiumes secundarios (D). 